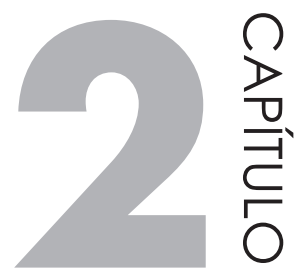

\title{
SISTEMAS DE UNIDADES
}

\subsection{SISTEMA MKSC}

Comprimento: metro (m)

Massa: quilograma $(\mathrm{kg})$

Tempo: segundo (s)

Carga elétrica: Coulomb (C).

O metro foi definido originalmente como a décima milionésima parte da distância do Polo ao Equador no meridiano de Paris. ${ }^{1}$ Posteriormente verificou-se que ele diferia $0,023 \%$ do valor pretendido (barra do metro-padrão).

Então, adotou-se como padrão o valor de $1.650 .763,73$ comprimentos de onda da luz emitida, no vácuo, pelo isótopo do criptônio $K r^{86}$, excitado por carga elétrica, na radiação vermelho alaranjada.

Assim: comprimento de onda $\lambda=6057,80211 \mathrm{~A}$

$$
1 \AA=10^{-10} \mathrm{~m}
$$

\footnotetext{
Ou seja $10^{-7}$ de um quadrante do meridiano terrestre, isto é, $1 / 4$ de uma circunferência passando pelos polos.
} 


$$
1.650 .763,73 \times 6057,80211 \times 10^{-10}=1,00 \mathrm{~m}
$$

Utiliza-se um interferômetro de Michelson (o mesmo utilizado na célebre experiência de MichelsonMorley).

Esquema:

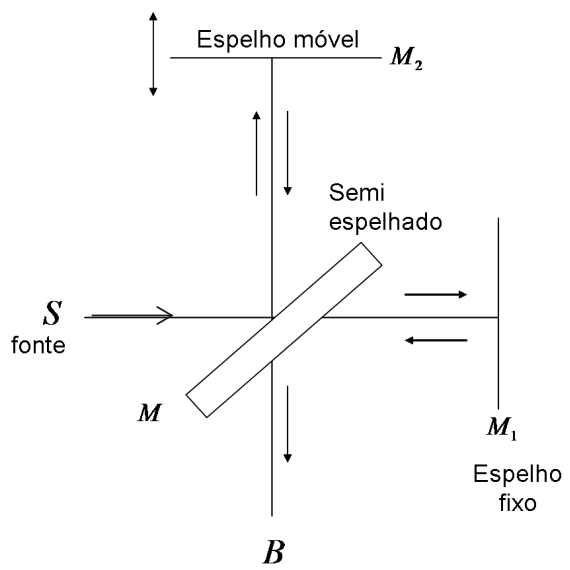

Formação das franjas

\subsubsection{Fendas múltiplas}

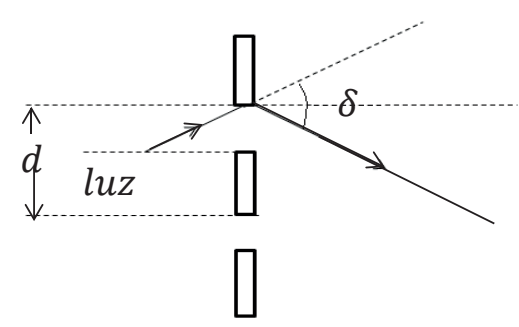

$$
\operatorname{sen} \frac{\delta}{2}=\frac{\mathrm{n} \lambda}{2 \mathrm{~d}}
$$

$\lambda=$ comprimento de onda

São fendas paralelas, de mesma largura, separadas por intervalos iguais. Esse dispositivo é denominado "Rede de difração". Sua primeira construção foi realizada em 1814 por Joseph von Fraunhofer (1787-1826). 
As redes de difração foram inicialmente montadas com fios metálicos muito finos, de 0,04 a 0,6 mm de diâmetro, separados por intervalos de $0,0528 \mathrm{~mm}$ a 0,6866 mm. Atualmente, são constituídas com uma lâmina de vidro onde se riscam, com diamante, uma grande quantidade de linhas retas igualmente intercaladas.

Cada fenda gera uma onda. As ondas paralelas se interferem, como as ondas do mar ou do som. Quando o som ricocheteia em uma parede, as interferências podem se aniquilar ou reforçar, e também provocar ecos. Algo semelhante acontece quando a luz se reflete nos espelhos do Interferômetro de Michelson (SEARS; STRATHERN).

$\mathrm{Na}$ interferência, formam-se franjas, isto é, zonas intercaladas de claro e escuro. As zonas claras são de reforço, e as escuras, de aniquilação das ondas. [Ver redes de difração de Fraunhofer (1787-1826)].

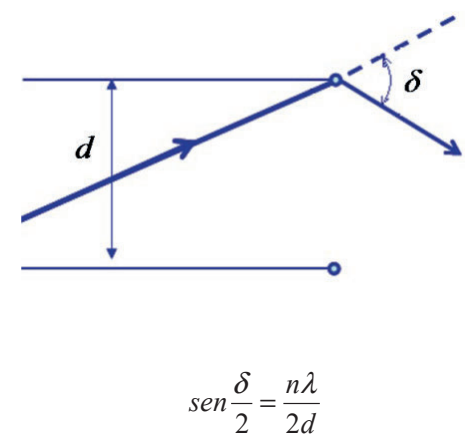

A rede de difração é formada pela lâmina de vidro onde se riscam, com diamante, um grande número de linhas retas igualmente intervaladas.

No interferômetro de Michelson, no lugar da lâmina de vidro com riscas, teremos a interferência devida aos trens de ondas refletidas em $M_{1}$ e $M_{2}$, que interferem em $M$ e, conforme o deslocamento de $M_{2}$, podem ser reforçados ou anulados, formando franjas de interferência, semelhantes a listras de zebra (SEARS).

Em nosso caso, $\delta=180^{\circ}, \operatorname{logo}, \lambda=\frac{2 d}{n}$.

\subsubsection{Exemplo ilustrativo}

Contam-se 792 franjas ao se deslocar o espelho $M_{2}$ de $0,233 \mathrm{~mm}$.

Qual é o comprimento da luz utilizada?

$$
\lambda=\frac{2 L}{N}=\frac{2 \times 0,233 \times 10^{7}}{792}=5883 \AA
$$


Quando o espelho $M_{2}$ é deslocado de uma distância $\lambda / 2$, a diferença do trajeto de luz variará de $\lambda$, e a figura de interferência sofrerá um deslocamento de uma franja.

A precisão alcançada com o metro padrão é de cerca de uma parte em $10^{7}$. Se esse erro ocorresse no ajuste da bússola giroscópica, poderia ocasionar um desvio de $1.600 \mathrm{~km}$ em um foguete lançado para a Lua.

As ondas luminosas têm comprimento de aproximadamente $5 \times 10^{-5} \mathrm{~cm}$, resultando em uma precisão de uma parte em $10^{9}$.

Originalmente, o quilograma foi definido como a massa de um bloco de platina mantido em Sèvres, nas proximidades de Paris.

Para fins práticos, a massa de $1 \mathrm{~kg}$ é 1.000 vezes igual à massa de $1 \mathrm{~cm}^{3}$ de água destilada à temperatura de $4^{\circ} \mathrm{C}$, condição na qual a densidade da água é máxima (ALONSO, FINN; HALLIDAY, RESNICK; ENC. BRITANNICA).

A fórmula da densidade é: $\rho=\frac{m}{V}$, ou seja, massa por unidade de volume.

Para fins práticos, um litro de água tem a massa de $1 \mathrm{~kg}$

$$
1 l=1 \mathrm{dm}^{3} \quad 1 \mathrm{dm}=10 \mathrm{~cm} \quad 1 \mathrm{~m}=100 \mathrm{~cm}
$$

Logo, $1 \mathrm{dm}^{3}=1000 \mathrm{~cm}^{3}$.

Qualquer fenômeno periódico que se repita no tempo pode servir para sua medição. Pode ser um pêndulo oscilante, uma mola espiral ou um cristal de quartzo.

Desde os tempos primitivos, a duração do dia tem sido usada como padrão de tempo.

O dia tem 24 h de 60 min cada, e cada min, 60 s. Logo, o dia tem: $24 \times 60 \times 60=86.400 \mathrm{~s}$.

Define-se o segundo $(S$ ) como a fração 1/86400 do dia solar médio e é denominado de "tempo universal" (TU).

Em 1956, o Congresso Internacional de Pesos e Medidas redefiniu o segundo considerando um período anual.

Devido às ações das marés, o período de rotação da Terra está aumentando gradualmente, por isso, tomou-se o ano de 1900, fornecendo o ano trópico (intervalo entre duas passagens sucessivas pelo equinócio vernal, em 21 de março de cada ano) com 365d 5h 48 min 45,97 seg = 31556925,9747 s

Assim, o segundo é definido então como a fração: 1/31556925,9747 do ano trópico de 1900, e essa unidade é denominada "tempo das efemérides" (TE). 


\subsubsection{Pêndulo simples}

Em 1581, Galileu entrou na Universidade de Pisa para estudar Medicina. Em certa ocasião, quando assistia a uma missa na Catedral de Pisa, observou uma lâmpada que balançava pendurada do teto por um longo fio. Ele notou que, independentemente da amplitude, o período de oscilação era constante. Ao chegar em casa, construiu um pêndulo com um pedaço de corda e um peso de chumbo. Ao realizar experiências com pesos e cordas de comprimentos diferentes, chegou à conclusão de que o período, para pequenas oscilações, dependia unicamente do comprimento do pêndulo.

Então, Galileu concebeu um tipo de pêndulo que podia ser usado para medir os batimentos cardíacos dos pacientes e o apresentou a alguns membros do departamento médico, que ficaram tão impressionados a ponto de roubar sua ideia (naquele tempo não havia registro de patentes). Apesar disso, o pulsilogium, como ficou conhecido, granjeou a Galileu certo renome local.

Posteriormente Christiaan Huygens, em 1656, foi o responsável por sua aplicação geral como controlador do tempo em relógios.

A grande virtude do pêndulo como medidor do tempo está no fato de que, para pequenos arcos de oscilação, seu período depende somente do comprimento do pêndulo e praticamente independe da extensão do arco.

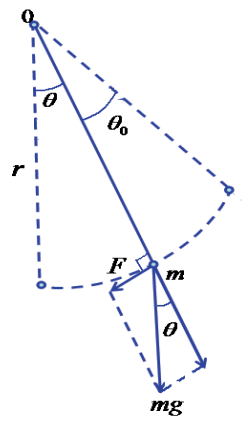

Sendo $F$ a força restauradora $F=-m g \operatorname{sen} \theta$

O sinal negativo indica que a sua direção é contrária ao crescimento do arco.

Para pequenas oscilações: $\operatorname{sen} \theta \cong \theta$

Logo: $F=-m g \theta$

O ângulo $\theta$ em radianos é medido como a razão entre o arco e o raio: ${ }^{2}$

Outro modo é medir os ângulos em graus, tomando como base o ângulo reto $90^{\circ}$. Como a circunferência tem quatro ângulos retos, tem-se: $4 \times 90^{\circ}=360^{\circ}$. Assim, a circunferência completa tem $360^{\circ}=2 \pi \mathrm{rad}$ 


$$
\theta=\frac{x}{r}
$$

Assim, a circunferência tem $2 \pi$ radianos.

Portanto: $F=-m g \frac{x}{r}=-\frac{m g}{r} x$

A força é proporcional ao deslocamento $x$.

\subsubsection{Movimento harmônico simples (MHS)}

Seja um corpo de massa $m$ deslizando sobre uma superfície lisa e ligado a uma mola.

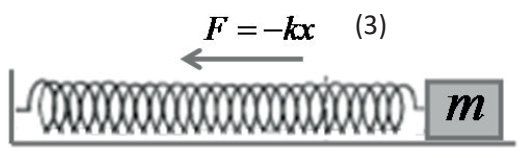

Distendemos a mola e soltamos o corpo, que tende a voltar à posição inicial, mas oscila entre as posições $-x \mathrm{e}+x$.

Chamamos $F$ de força restauradora, ou força elástica (devido às propriedades elásticas da mola). A fórmula $F=-k x$ é conhecida como Lei de Hooke, de autoria de Robert Hooke (1635-1703), que descreve a deformação ${ }^{3}$ elástica dos corpos. A lei foi descoberta em 1660 e publicada somente 16 anos depois.

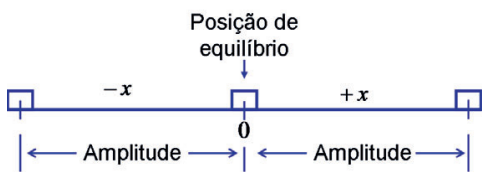

$$
F=-k x=m a \therefore a=-\frac{k}{m} x
$$

Onde $a$ é aceleração e a Força $F$ é massa vezes aceleração (ver seções 4.3 e 4.4)

$k$ é chamada de constante elástica

Consideremos o ponto Q movendo-se em trajetória circular com velocidade angular constante $\omega$ $(\mathrm{rad} / \mathrm{seg}): \omega=\frac{d \theta}{d t}$.

Ver Capítulo 3, "Elasticidade". 
$P$ é a projeção de $Q$ no eixo dos $x$ e $P$ se move para frente e para trás em movimento harmônico simples, como veremos.

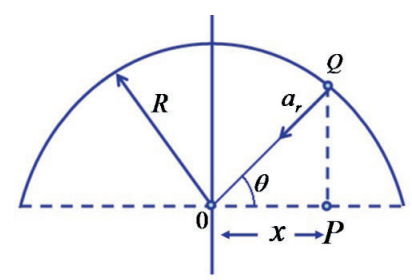

Consideremos somente a cinemática do movimento $P$, isto é, não consideramos a força que o provoca. A aceleração de $P$ será: $a=-a_{r} \cos \theta$, sendo $a_{\mathrm{r}}$ aceleração radial ou centrípeta.

Para calcular $a_{r}$ :
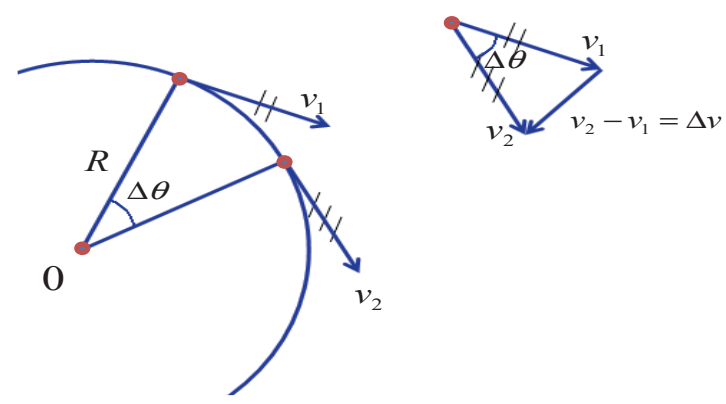

$a_{r}=\lim _{\Delta t \rightarrow 0} \frac{\Delta v}{\Delta t} \quad \Delta \theta=\frac{\Delta v}{v} \quad$ ou $\quad \Delta v=v \Delta \theta$ (aproximadamente)

$\frac{\Delta v}{\Delta t}=v \frac{\Delta \theta}{\Delta t}($ aproximadamente $)$

Quando $\Delta t \rightarrow 0$, a aproximação torna-se exata:

$$
\lim _{\Delta t \rightarrow 0} \frac{\Delta v}{\Delta t}=v \lim _{\Delta t \rightarrow 0} \frac{\Delta \theta}{\Delta t} \quad \text { Porém: } \omega=\lim _{\Delta t \rightarrow 0} \frac{\Delta \theta}{\Delta t}
$$

Logo: $a_{r}=v \omega$ 
Porém, $v=R \omega$

Deduzindo da Equação (1), derivando em t:

$$
\begin{aligned}
& \frac{d \theta}{d t}=\frac{1}{R} \frac{d x}{d t}, R \text { é constante, } \omega=\frac{d \theta}{d t} \text { e } v=\frac{d x}{d t} \\
& \therefore a_{r}=v \omega=R \omega^{2}=\frac{v^{2}}{R}
\end{aligned}
$$

Voltando ao movimento harmônico simples (MHS): $a=-a_{r} \cos \theta \quad \therefore a=-\omega^{2} R \cos \theta$, sendo em nosso caso, $R$ amplitude máxima: $\quad x=R \cos \theta$

Logo: $\quad a=-\omega^{2} x \quad$ Como $\omega$ é constante, $a$ só depende de $x$.

Comparando com $a=-\frac{k}{m} x$, ver Equação (3a).

Então: $\omega^{2}=\frac{k}{m} \quad$ Mas: $\omega=\frac{2 \pi}{T}$

Sendo T o período de uma volta.

O ângulo de uma volta é $2 \pi$ rad, ou período, e T é o tempo gasto em uma volta ou período, resultando $\omega$ em rad por seg, velocidade angular.

$$
\text { Logo: } T=\frac{2 \pi}{\omega} \quad \therefore T=2 \pi \sqrt{\frac{m}{k}}
$$

Outra forma de resolver é:

Como: $a=\frac{d^{2} x}{d t^{2}}$

Portanto: $\frac{d^{2} x}{d t^{2}}+\frac{k}{m} x=0$

Então: $\frac{d^{2} x}{d t^{2}}=-\frac{k}{m} x$
Temos da equação: $a=-\frac{k}{m} x$

É uma equação diferencial de $2^{\mathrm{a}}$ ordem.

É uma função cuja derivada de segunda ordem em relação ao tempo seja igual à própria função, com sinal oposto e multiplicado por uma constante $\mathrm{k} / \mathrm{m}$. As funções seno e cosseno satisfazem essas condições. Assim:

$$
\frac{d}{d t} \cos t=-\operatorname{sen} t \quad \frac{d^{2}}{d t^{2}} \cos t=-\frac{d}{d t} \operatorname{sen} t=-\cos t
$$

Essa propriedade não é afetada se a função cosseno for multiplicada pela constante $R$. Levando em consideração $\theta=\omega t$, sendo $\omega$ constante: $\mathrm{x}=\mathrm{R} \cos \omega \mathrm{t}$. 


$$
\frac{d x}{d t}=-\omega R \operatorname{sen} \omega t \quad \frac{d^{2} x}{d t^{2}}=-\omega^{2} R \cos \omega t
$$

Substituindo na equação: $\frac{d^{2} x}{d t^{2}}=-\frac{k}{m} x$

Teremos: $-\omega^{2} R \cos \omega t=-\frac{k}{m} R \cos \omega t$

Conclui-se que: $\omega^{2}=\frac{k}{m}$

Significado físico da constante $\omega$.

Se o tempo $t$ for aumentado de $2 \pi / \omega$, a função se torna:

$x=R \cos \omega(t+2 \pi / \omega)=R \cos (\omega t+2 \pi)=R \cos \omega t$

Isto é, a função é periódica e se repete após o intervalo de tempo $2 \pi / \omega$, que é denominado de período $T$.

Como $\omega^{2}=\frac{k}{m}$, ver Equação $(3 \mathrm{c})$,

$\mathrm{T}=\frac{2 \pi}{\omega}=2 \pi \sqrt{\frac{\mathrm{m}}{\mathrm{k}}}$

[Ver Seção 5.22, Equação (0)]

e $\omega=\frac{2 \pi}{T}$, que é uma velocidade angular $(\mathrm{rad} / \mathrm{seg})$.

\subsubsection{Pêndulo simples}

Retomando a Equação 2: $F=-\frac{m g}{r} x$, podemos fazer a análise dinâmica desse MHS.

Vemos que obedece à Lei de Hooke: $F=-k x$, Equação (3).

Comparando as duas equações: $k=\frac{m g}{r}$, introduzindo na Equação (4b1),

$$
\therefore \frac{\mathrm{d}^{2} \mathrm{x}}{\mathrm{dt}^{2}}=-\frac{\mathrm{mg} / \mathrm{r}}{\mathrm{m}} \mathrm{x}=-\frac{\mathrm{g}}{\mathrm{r}} \quad \text { ou }
$$

$\frac{\mathrm{d}^{2} \theta}{\mathrm{dt}^{2}}=-\frac{\mathrm{g}}{\mathrm{r}} \theta(6)$, pois $\mathrm{x}=\mathrm{r} \theta$, ver Equação (1) e Anexo 6, "Resolução da equação da onda", Equação (9). 
Substituindo na equação do período: $T=2 \pi \sqrt{\frac{m}{k}} \quad$ [Equações (4) e (5)]

$$
T=2 \pi \sqrt{\frac{m}{m g / r}} \quad \therefore T=2 \pi \sqrt{\frac{r}{g}} \quad \therefore \frac{\mathrm{d}^{2} \mathrm{x}}{\mathrm{dt}^{2}}=\frac{\mathrm{mg} / \mathrm{r}}{\mathrm{m}} \mathrm{x}=-(\mathrm{g} / \mathrm{r}) \mathrm{x} \quad \text { ou }
$$

$\mathrm{d}^{2} \theta / \mathrm{dt}^{2}=-(\mathrm{g} / \mathrm{r}) \theta \quad$ (6), pois $\mathrm{x}=\mathrm{r} \theta$, ver Equação (1) e também Anexo 6, "Resolução da equação da onda", Equação (9).

Vemos que para pequenas oscilações (desde que $\operatorname{sen} \theta \cong \theta$ ). Té independente de $m$. Depende somente de $r$. É um método conveniente para medir a aceleração da gravidade $g$ (conhecendo $T$ e $r$ ).

Quando a amplitude de oscilação não for pequena, usamos: $r \frac{d^{2} \theta}{d t^{2}}=-g \operatorname{sen} \theta$

Integrando em relação a $t: \quad\left(\frac{d \theta}{d t}\right)^{2}=\frac{2 g}{r}(\cos \theta-\cos \alpha)$

Integrando novamente em $t$ :

$$
T=\sqrt{\frac{2 r}{g}} \int_{-a}^{a} \frac{d \theta}{\sqrt{\cos \theta-\cos \alpha}}=\sqrt{\frac{r}{g}} \int_{-a}^{a} \frac{d \theta}{\sqrt{\operatorname{sen}^{2} \frac{\alpha}{2}-\operatorname{sen}^{2} \frac{\theta}{2}}}
$$

(Integral elíptica)

$\alpha$, amplitude de oscilação do pêndulo, posição angular para $t=0$ com velocidade 0 .

Desenvolvendo pelo teorema do binômio, e depois integrando termo por termo:

$$
T=2 \pi \sqrt{\frac{r}{g}}\left(1+\frac{1}{2^{2}} \cdot \operatorname{sen}^{2} \frac{\theta_{m}}{2}+\frac{1}{2^{2}} \frac{3^{2}}{4^{2}} \operatorname{sen}^{4} \frac{\theta_{m}}{2}+\ldots\right)
$$

$\theta_{m}$ deslocamento angular máximo (COURANT; YAVORSKI; HÜTTE).

Somente para amplitudes muito grandes é que o pêndulo difere de $T_{0}=2 \pi \sqrt{\frac{r}{g}}$

Para pequenas amplitudes é suficiente tomar apenas o primeiro termo de correção e pode-se substituir $\operatorname{sen} \frac{1}{2} \theta_{0}$ por $\frac{1}{2} \theta_{0}$, resultando em: $T=2 \pi \sqrt{\frac{r}{g}}\left(1+\frac{1}{16} \theta_{0}^{2}\right)$

$\theta_{0}$ em rad 
É suficiente para a maioria das situações práticas. De fato, o termo $\theta_{0}^{2} / 16$ contribui com menos de $1 \%$ para amplitudes menores que $23^{\circ}$.

Para maior exatidão, o período pode ser calculado com o grau de precisão necessário, tomando-se a quantidade suficiente de termos da série.

Quando $\theta_{m}=15^{\circ}$, o período mais exato difere do aproximado em menos de $0,5 \%$.

\subsubsection{Pêndulo de torção}

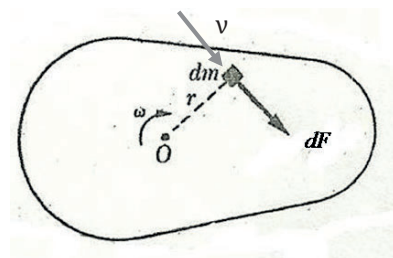

Conjugado ou torque elementar: $d \mathrm{C}=r d F$ (7a) [Momento da força elementar; ver Equação (2), Capítulo 3, "Elasticidade"].

Porém, pela Segunda Lei de Newton: $d F=\frac{d}{d t}(v d m)$ e $v=\omega \mathrm{r} \quad \therefore d F=r d m \frac{d \omega}{d t}$

Nesse caso, a massa $d m$ e o raio $r$ não variam com o tempo.

$$
\alpha=\frac{d \omega}{d t}
$$

Logo: $d \mathrm{C}=r^{2} d m \alpha$

$\alpha=$ aceleração angular

A quantidade: $d I=r^{2} d m$ chama-se momento de inércia elementar.

Integrando para o corpo todo, teremos o torque total: $\mathrm{C}=\int \alpha r^{2} d m$

$$
\therefore \mathrm{C}=\alpha \int \mathrm{r}^{2} \mathrm{dm}=\frac{\mathrm{d} \omega}{\mathrm{dt}} \int \mathrm{dI}
$$

A expressão geral do conjugado será: $\mathrm{C}=\frac{d}{d t}(I \omega)$, semelhante a $F=\frac{d}{d t}(m v)$.

Considerando a quantidade de movimento angular do elemento: $v r d m=\omega r^{2} \mathrm{dm}=\mathrm{dL}$.

A quantidade de movimento angular do total do corpo será: $\int \omega \mathrm{r}^{2} \mathrm{dm}=\omega \int \mathrm{r}^{2} \mathrm{dm}$. 
Como vimos, $I=\int \mathrm{r}^{2} \mathrm{dm}$, é o momento de inércia do corpo. Então, a quantidade de movimento angular pode ser definida como: $L=I \omega$.

$$
C=\frac{d}{d t}(I \omega)=\frac{d}{d t}\left[\int \mathrm{r}^{2} \mathrm{dm}(\omega)\right]=\frac{d \omega}{d t} \int \mathrm{r}^{2} \mathrm{dm}=\alpha I, \text { para } I \text { constante no tempo, como vimos }
$$
anteriormente.

$$
\text { Logo: } \mathrm{C}=I \alpha \quad \text { (para } I \text { constante), pois } \alpha=\frac{d \omega}{d t} .
$$

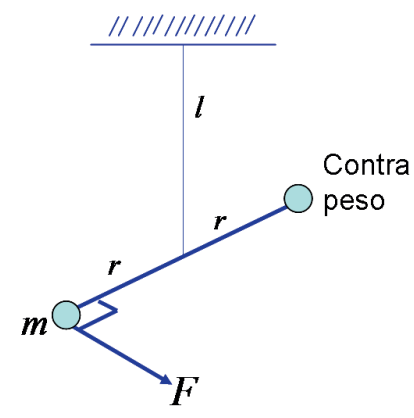

A balança de torção de Cavendish, utilizada para experiências com massas e cargas elétricas, é constituída de um fio de fibra ou metálico, de comprimento $l$ que sustenta uma haste de comprimento $2 r$, tendo em uma extremidade um corpo de prova (massa ou carga elétrica) e, na outra extremidade, um contrapeso. O corpo de prova fica sujeito a uma força de atração ou repulsão $F$. A haste é defletida de um ângulo $\theta$ na horizontal, provocando no fio uma torção que deve ser contrabalançada.

Essa deflexão é provocada pelo torque (momento):

$\mathrm{C}=-K \boldsymbol{\theta}(8) \quad$ [Ver Capítulo 3, "Elasticidade”, Equação (3)]

$K$ (kapa) é o módulo de torção do fio, que depende do material do fio.

Momento $\mathrm{C}=F \cdot r$

A fórmula é válida para pequenas deflexões.

Como $\mathrm{C}=I \alpha=I \frac{d^{2} \theta}{d t^{2}}$, temos $\frac{d^{2} \theta}{d t^{2}}=-\frac{K}{I} \theta$

Para $\quad \theta=\frac{x}{r}$, sendo $x$ o deslocamento do corpo de prova 


$$
\begin{gathered}
\frac{d^{2}}{d t^{2}} \theta=\frac{d^{2}}{d t^{2}}\left(\frac{x}{r}\right)=-\frac{K}{I}\left(\frac{x}{r}\right), \text { como } r \text { é constante, } \frac{d^{2} x}{d t^{2}}=-\frac{K}{I} x \\
F=-k x \quad \text { e } \quad a=-\frac{k}{m} x
\end{gathered}
$$

Comparando com a Equação (3a), vemos que obedece à Lei de Hooke.

Não contrabalançando a torção, o corpo de prova vai oscilar com um período como na Equação (4): $T=2 \pi \sqrt{\frac{m}{k}}$

Em nosso caso: $\therefore T=2 \pi \sqrt{\frac{I}{K}}$

Conhecendo $K$ e medindo $T$, pode-se determinar o $I$ de qualquer corpo rígido em relação ao eixo de rotação. Ou conhecendo $I$ e medindo $T$, pode-se determinar $K$.

Muitos instrumentos envolvem oscilações de torção, como o galvanômetro e a balança de torção.

O volante de um relógio é um exemplo de movimento harmônico angular. O torque restaurador é fornecido por uma mola espiral (“cabelo").

\subsubsection{Quartzo}
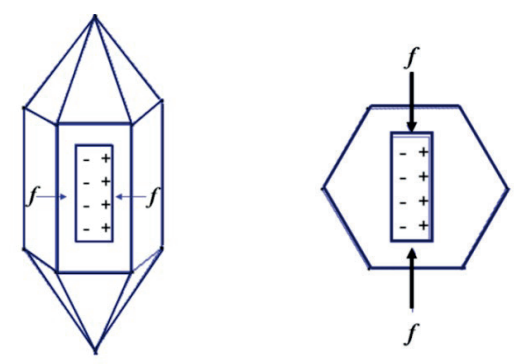

O cristal de quartzo tem a forma de um prisma hexagonal, terminado por duas pirâmides hexagonais.

De duas em duas, as arestas laterais são terminadas por facetas, que faltam nas arestas intermediárias, resultando uma dissimetria particular.

Suponhamos ter cortado um prisma reto de base retangular, como mostra a Figura, com faces paralelas aos eixos principais do cristal.

Jacques e Pierre Curie descobriram, em 1882, que esse prisma é piezoelétrico, isto é, que pode se eletrizar por compressão ou tração, e que suas faces opostas ficam com cargas de sinais diferentes. 
Reciprocamente, o fenômeno é reversível, isto é, ao eletrizar as referidas faces, o prisma se alongará ou encurtará.

Como aplicação, utiliza-se para medidas elétricas e substitui o galvanômetro. Também produz ultrassons. Um sistema oscilante de radiofrequência, produzindo com frequência de $100.000 \mathrm{~Hz}$, carrega alternadamente as duas faces de uma lâmina de quartzo, produzindo contrações e dilatações de $100.000 \mathrm{~Hz}$, fazendo vibrar por ressonância um tubo sonoro.

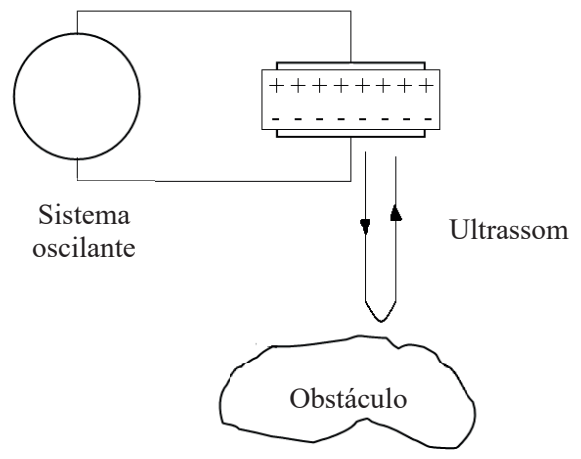

O ultrassom será produzido, propagando-se na água a $1500 \mathrm{~m} / \mathrm{s}$, altamente direcional e sem difração.

As vibrações retornam e o próprio cristal atua como receptor, emitindo corrente elétrica de sinal fraco, que é amplificado através da grade de uma válvula triodo ou transistor. As ondas são ouvidas e registradas. Serve para detectar submarinos, nivelamento do fundo do mar, icebergs, navios submersos e outros obstáculos (LEMOINE, GUYOT).

Atualmente, é muito utilizado na medição de tempo, em relógios e cronômetros, ao proporcionar uma frequência constante. Este assunto será retomado oportunamente.

O relógio mais preciso inventado até hoje é o atômico, por basear-se na transição entre dois estados quânticos do átomo de $C s^{133}$, em que há absorção ou emissão de radiação eletromagnética de uma frequência bem definida. ${ }^{4}$ Um exemplo desse relógio é o que está instalado no National Physical Laboratory em Teddington, próximo de Londres. (7)

Frequência $=9.192 .631 .770 \mathrm{oscil} / \mathrm{seg}$

Com isso, o momento magnético dos átomos de $C s^{133}$ é alterado, podendo ser convenientemente defletido por campos magnéticos oscilantes (mesma frequência da transição). 
C, a quarta letra de MKSC, é a unidade de carga elétrica Coulomb, que será definida oportunamente. No momento, podemos dizer que é, em valor absoluto, igual ao conteúdo de carga negativa de $6,2418 \times 10^{18}$ elétrons, ou de igual número de carga positiva de prótons. ${ }^{5}$

Em engenharia é comum utilizar-se do sistema $M K S$ técnico, em que a força constitui uma grandeza fundamental em lugar da massa, que passa a ser grandeza derivada. Nesse caso, a força será medida em $k g f$.

\subsection{SISTEMA MKSA}

Comprimento: metro (m)

Massa: quilograma $(\mathrm{kg})$

Tempo: segundo (s)

Corrente elétrica: Ampère $(A)$.

Também chamado de Sistema Internacional (SI) de unidades, foi adotado na $11^{\text {a }}$ Conferencia Geral de Pesos e Medidas, em Paris (1960), e, nesse caso, o Ampère (A) é escolhido por ser mais fácil de medir.

O Coulomb é então definido como a quantidade de carga elétrica que atravessa uma secção de um condutor durante $1 \mathrm{~s}$, quando a corrente que o percorre é de $1 \mathrm{~A}$.

\subsection{SISTEMA CGS (SISTEMA GAUSSIANO)}

É muito popular nos trabalhos científicos, nele tem-se:

Comprimento: centímetro $(\mathrm{cm})$

Massa: grama (g)

Tempo: segundo (s).

Ver a definição apresentada na página inicial do capítulo 5 . 
